\title{
Terahertz On-Chip Antenna Based on Metasurface and SIW with Stacked Layers of Resonators on GaAs Substrate
}

\author{
Mohammad Alibakhshikenari ${ }^{1 *}$, Bal S. Virdee ${ }^{2}$, Chan H. See ${ }^{3,4}$, Sam Jeffery Fishlock ${ }^{5}$, Navneet Soin ${ }^{5}$, James \\ McLaughlin $^{5}$, Raed A. Abd-Alhameed ${ }^{6}$, and Ernesto Limiti ${ }^{1}$ \\ ${ }^{1}$ Electronic Engineering Department, University of Rome "Tor Vergata", Via del Politecnico 1, 00133, Rome, ITALY \\ ${ }^{2}$ London Metropolitan University, Center for Communications Technology \& Mathematics, School of Computing \& Digital Media, \\ London N7 8DB, UK \\ ${ }^{3}$ School of Eng. \& the Built Environment, Edinburgh Napier University, 10 Colinton Rd., Edinburgh, EH10 5DT, UK \\ ${ }^{4}$ School of Engineering, University of Bolton, Deane Road, Bolton, BL3 5AB, UK \\ ${ }^{5}$ School of Engineering, Nanotechnology and Integrated Bioengineering Centre (NIBEC), University of Ulster, Newtownabbey, \\ BT37 0QB, Northern Ireland, UK \\ ${ }^{6}$ School of Electrical Engineering \& Computer Science, University of Bradford, Bradford, BD7 1DP, UK \\ *alibakhshikenari@ing.uniroma2.it
}

\begin{abstract}
This paper presents a $100 \mu \mathrm{m}$ GaAs-based 0.45$0.50 \mathrm{THz}$ on-chip antenna based on metasurface and substrate integrated waveguide (SIW) technologies to realize a high-performance antenna. The antenna design consists of $2 \times 4$ array of circular slot resonators embedded in rectangular ground-plane segments in a horizontal arrangement. The ground-plane segments are separated from each other by a narrow channel to create a coplanar waveguide which is used to excite the structure. This antenna structure, which is constructed on GaAs substrate, reduces substrate loss and surface waves. In addition, the metasurface essentially enlarges the effective aperture area of the antenna to enhance the gain and radiation efficiency of the antenna. The dimensions of the metasurface on-chip antenna is $0.8 \times 0.8 \times 0.13 \mathrm{~mm}^{3}$. The antenna exhibits an average gain and efficiency of $6.9 \mathrm{dBi}$ and $61.82 \%$, respectively, which makes it a promising candidate for packaging in terahertz components.
\end{abstract}

Keywords: On-chip antenna, metasurface, terahertz, SIW, GaAs layer, resonators, high performances.

\section{INTRODUCTION}

Antennas for on-chip applications need to be planar as they need to be fabricated using the same integrated circuit processing. Low resistivity substrate $(10-20 \Omega \mathrm{cm})$ is a common choice for the fabrication of CMOS circuits which is necessity to overcome the latch-up issue. This type of substrate however greatly limits the efficiency of planar antennas resulting from high ohmic and dielectric loss in the underlying silicon substrate. A dipole antenna fabricated on a $10 \Omega \mathrm{cm}$ silicon substrate has an efficiency of just $10 \%[1,2]$.

In this paper, we have proposed two applicable approaches to overcome the limitations on bandwidth, gain and efficiency of the on-chip antennas for integration in terahertz integrated circuits. The proposed technique reduces the substrate loss and suppresses surface waves. This results in improved performance parameters of the onchip antenna. The proposed methods are based on 2dimensional metamaterials, which are commonly referred to as metasurface, and substrate integrated waveguide (SIW).

\section{Proposed Methods to OVERCome the Limitations ON BANDWIDTH, GAIN AND EFFICIENCY}

The proposed on-chip antenna comprises of a $2 \times 4$ array of a series of circular slot resonators implemented in the rectangular ground-plane on top of a Gallium Arsenide (GaAs) substrate with a ground-plane on its lower side, as shown in Fig.1. The horizontal slot resonators across the upper rectangular ground-plane act like miniature resonators that radiate energy at $\mathrm{THz}$ band. The $2 \times 4$ ground-plane sections on the GaAs is framed by a metallic strip that is punctuated at its periphery with metallic viasholes. The Gallium Arsenide (GaAs) substrate with thickness of $100 \mu \mathrm{m}$, dielectric constant of 12.94, and losstangent of 0.006 .

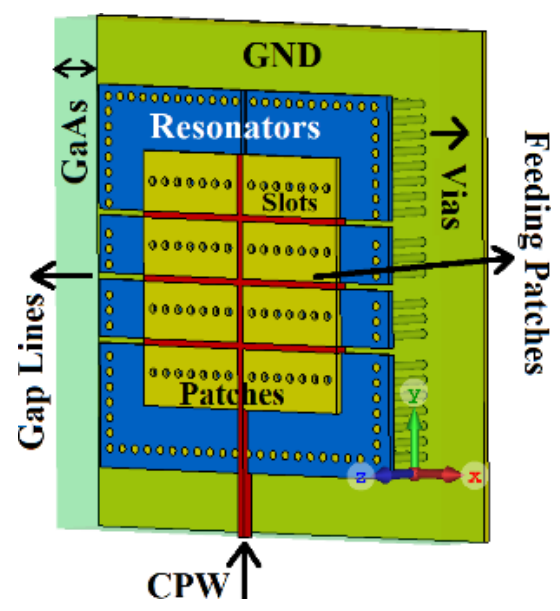

Fig.1. The proposed on-chip antenna incorporating metamaterial and SIW technologies.

The resonators effectively increase the impedance match and impedance bandwidth of the antenna in the terahertz band. In addition, to decrease the substrate loss and surface waves the metallic via-holes have actualized on the resonators in a substrate integrated waveguide configuration. The metallic vias are connected to the ground-plane through GaAs substrate. The via-holes act as shunt left-handed (LH) inductors $\left(L_{L}\right)$, which is one of the components in the metamaterial structures [3, 4]. By optimizing the via diameters and the space between them 
the we can tune $L_{L}$ which leads to further reduction in surface waves and substrate loss. The consequence of this is improved impedance matching, impedance bandwidth, and radiation properties. The gap between the metal slots play the role of the series LH capacitances $\left(C_{L}\right)$. By optimizing the number of the slots, their dimensions, and the space between them the value of $C_{L}$ can be tuned. The shunt $L_{L}$ and series $C_{L}$ transform the structure to exhibit metamaterial properties.

With the proposed configuration the excitation of the structure is based on coplanar waveguide (CPW), as shown in Fig.2. The feed-line in the narrow gaps separating the rectangular ground-planes is used to couple electromagnetic energy to each of the resonators embedded in the rectangular ground-planes. This configuration reduces the substrate loss and surface waves. The structural parameters of the proposed on-chip antenna are given in Table I. The on-chip antenna has a very compact foot print area of $0.8 \times 0.8 \times 0.13 \mathrm{~mm}^{3}$.
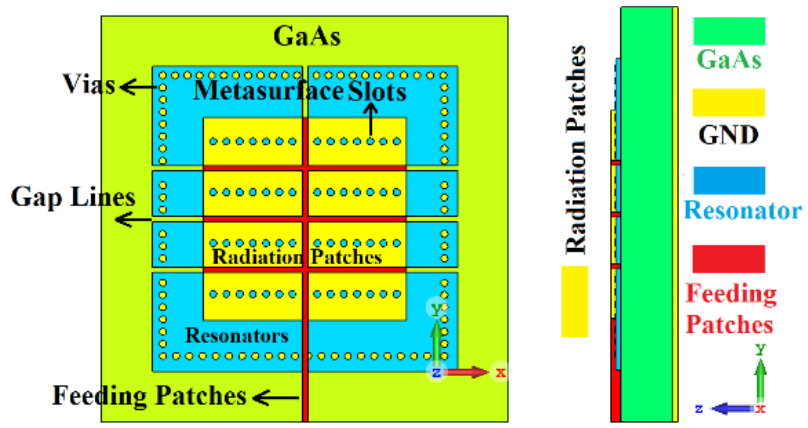

Fig.2. Top and side views of the proposed on-chip antenna showing the gap lines and feeding mechanism.

The reflection coefficient of the proposed on-chip antenna in Fig. 3 shows that it covers a wide frequency bandwidth from $0.45-0.5 \mathrm{THz}$ for $\mathrm{S}_{11} \leq-15 \mathrm{~dB}$, which corresponds to a fractional bandwidth of $10.52 \%$. The gain and radiation efficiency response over this frequency band in Fig. 4 shows the proposed antenna to have an average gain and efficiency of $6.9 \mathrm{dBi}$ and $61.82 \%$, respectively. The results confirm that with the proposed technique using metasurface and SIW technologies to realize an on-chip antenna provides high performance which is essential for applications in terahertz integrated-circuits.

TABLE I. STRUCTURAL PARAMETERS

\begin{tabular}{|c|c|}
\hline On-chip antenna size & $0.8 \times 0.8 \times 0.13 \mathrm{~mm}^{3}$ \\
\hline Occupied area by resonators & $0.6 \times 0.6 \times 0.01 \mathrm{~mm}^{3}$ \\
\hline Occupied area by patches & $0.4 \times 0.4 \times 0.01 \mathrm{~mm}^{3}$ \\
\hline Number of slots in each patch & 7 \\
\hline Slot's diameters & $7 \mu \mathrm{m}$ \\
\hline Slot's spacing & $26 \mu \mathrm{m}$ \\
\hline Via's diameters & $7 \mu \mathrm{m}$ \\
\hline Via's spacing & $26 \mu \mathrm{m}$ \\
\hline Gap-line's width & $10 \mu \mathrm{m}$ \\
\hline Thickness of GaAs & $100 \mu \mathrm{m}$ \\
\hline
\end{tabular}

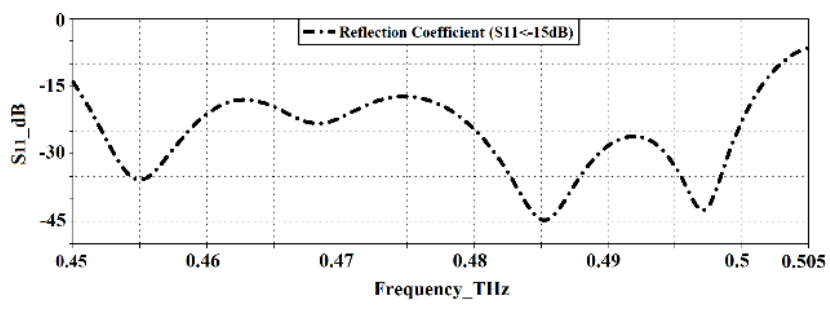

Fig.3. Reflection coefficient response of the proposed on-chip antenna.

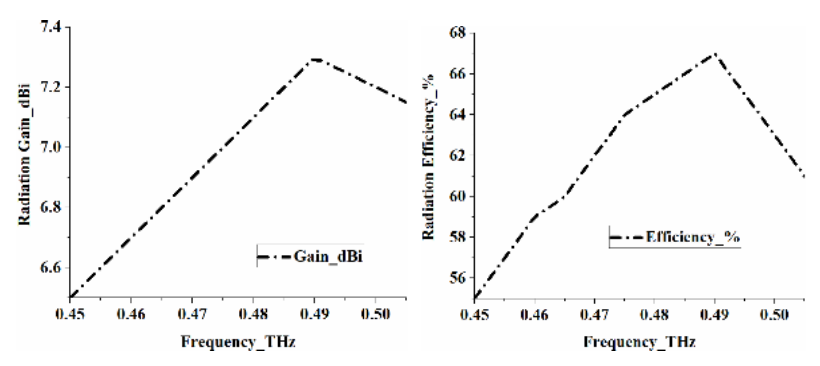

Fig.4. Radiation gain and efficiency as a function of frequency.

\section{CONCLUSION}

An effective antenna design is described for on-chip application operating in the terahertz frequency band. The design antenna employing metasurface and SIW technologies enhances the miniature antenna's gain and radiation characteristics. The antenna is constructed on a GaAs substrate and is composed of $2 \times 4$ array of circular resonator slots embedded in rectangular ground-plane, which are excited using coplanar waveguide arrangement to reduce substrate loss and surface waves.

\section{ACKNOWLEDGEMENTS}

This work is partially supported by innovation programme under grant agreement H2020-MSCA-ITN-2016 SECRET-722424 and the financial support from the UK Engineering and Physical Sciences Research Council (EPSRC) under grant EP/E022936/1.

\section{REFERENCES}

[1] Wonseok Choe and Jinho Jeong, "Broadband THz CMOS On-chip Antenna Using Stacked Resonators", 2017 IEEE International Symposium on Radio-Frequency Integration Technology (RFIT), pp.208210, 30 Aug.-1 Sept. 2017, Seoul, South Korea.

[2] Paolo Nenzi, Volha Varlamava, Frank Silvio Marzano, Fabrizio Palma and Marco Balucani, "U-Helix: On-Chip Short Conical Antenna", 2013 7th European Conference on Antennas and Propagation (EuCAP), pp.1289-1293, 8-12 April 2013, Gothenburg, Sweden.

[3] M. Alibakhshikenari, B. S. Virdee, A. Ali, and E. Limiti, "Miniaturized Planar-Patch Antenna Based on Metamaterial L-shaped Unit-Cells for Broadband Portable Microwave Devices and Multiband Wireless Communication Systems" IET Microwaves, Antennas \& Propagation, Volume 12, Issue 7, 13 June 2018, p. 1080 - 1086.

[4] M. Alibakhshi-Kenari, M. Naser-Moghadasi, R. A. Sadeghzadeh, B S. Virdee and E. Limiti, "Periodic Array of Complementary Artificial Magnetic Conductor Metamaterials-Based Multiband Antennas for Broadband Wireless Transceivers" IET Microwaves, Antennas \& Propagation, Volume 10, Issue 15, 10 December 2016, p. 1682 - 1691. 